\title{
On the projections of the multifractal packing dimension for $q>1$
}

\author{
Bilel Selmi ${ }^{1}$
}

Received: 8 April 2019 / Accepted: 12 November 2019 / Published online: 22 November 2019 (c) Fondazione Annali di Matematica Pura ed Applicata and Springer-Verlag GmbH Germany, part of Springer Nature 2019

\begin{abstract}
The aim of this article is to study the behavior of the multifractal packing function $B_{\mu}(q)$ under projections in Euclidean space for $q>1$. We show that $B_{\mu}(q)$ is preserved under almost every orthogonal projection. As an application, we study the multifractal analysis of the projections of a measure. In particular, we obtain general results for the multifractal analysis of the orthogonal projections on $m$-dimensional linear subspaces of a measure $\mu$ satisfying the multifractal formalism.
\end{abstract}

Keywords Hausdorff dimension · Packing dimension · Projection · Multifractal analysis

Mathematics Subject Classification $28 \mathrm{~A} 20 \cdot 28 \mathrm{~A} 80$

\section{Introduction and statement of the results}

The notion of singularity exponents or spectrum and generalized dimensions are the major components of the multifractal analysis. They were introduced with a view of characterizing the geometry of measure and to be linked with the multifractal spectrum which is the map which affects the Hausdorff or packing dimension of the iso-Hölder set

$$
E_{\mu}(\alpha)=\left\{x \in \operatorname{supp} \mu ; \lim _{r \rightarrow 0} \frac{\log (\mu B(x, r))}{\log r}=\alpha\right\}
$$

for a given $\alpha \geq 0$ and $\operatorname{supp} \mu$ is the topological support of probability measure $\mu$ on $\mathbb{R}^{n}$; $B(x, r)$ is the closed ball of center $x$ and radius $r$. It unifies the multifractal spectra to the multifractal packing function $B_{\mu}(q)$ via the Legendre transform [5,26], i.e.,

$$
\operatorname{dim}_{H}\left(E_{\mu}(\alpha)\right)=\inf _{q \in \mathbb{R}}\left\{q \alpha+B_{\mu}(q)\right\}
$$

Bilel Selmi

bilel.selmi@fsm.rnu.tn

1 Analysis, Probability and Fractals Laboratory LR18ES17, Department of Mathematics, Faculty of Sciences of Monastir, University of Monastir, 5000 Monastir, Tunisia 
There has been a great interest in understanding the fractal dimensions of projections of the iso-Hölder sets and measures. Recently, the projectional behavior of dimensions and multifractal spectra of sets and measures have generated a large interest in the mathematical literature $[3,7,14,15,18-20,29,30]$. The first significant work in this area was the result of Marstrand [22] who proved a well-known theorem according to which the Hausdorff dimension of a planar set is preserved under orthogonal projections. This result was later generalized to higher dimensions by Kaufman [21], Mattila [23] and Hu and Taylor [16], and they obtain similar results for the Hausdorff dimension of a measure.

Let us mention that Falconer and Mattila [12] and Falconer and Howroyd [11] have proved that the packing dimension of the projected set or measure will be the same for almost all projections. However, despite these substantial advances for fractal sets, only very little is known about the multifractal structure of projections of measures, except a paper by O'Neil [28]. Later, in [2] Barral and Bhouri studied the multifractal analysis of the orthogonal projections on $m$-dimensional linear subspaces of singular measures on $\mathbb{R}^{n}$ satisfying the multifractal formalism. The result of O'Neil was later generalized by Selmi et al. [7-9,32,33].

O'Neil [28] has compared the generalized Hausdorff and packing dimensions of a set $E$ of $\mathbb{R}^{n}$ with respect to a measure $\mu$ with those of their projections onto $m$-dimensional subspaces. More specifically, given a compactly supported Borel probability measure $\mu$ on $\mathbb{R}^{n}$ and $q \in \mathbb{R}$, let $B_{\mu}(q)$ be the multifractal packing function of supp $\mu$. Then, we have $B_{\mu_{V}}(q) \leq B_{\mu}(q)$ for all $q \leq 1$ and all $m$-dimensional linear subspaces $V$. Then, what can be said about the multifractal packing function and its projection onto a lower-dimensional linear subspace for $q>1$ ? The goal of this work is giving an answer to this question. We are interested in knowing whether or not this property is preserved after orthogonal projections on $\gamma_{n, m}$-almost every linear $m$-dimensional subspace for $q>1$, where $\gamma_{n, m}$ is the uniform measure on $G_{n, m}$, the set of linear $m$-dimensional subspaces of $\mathbb{R}^{n}$ endowed with its natural structure of a compact metric space (see [24]).

In the present paper, we pursue those kinds of studies and we consider the multifractal formalism developed in [28]. The aims of this study are twofold: First, the behavior of the packing dimensions $B_{\mu}(q)$ under projection. In particular, we show that $B_{\mu}(q)$ is preserved under $\gamma_{n, m}$-almost every orthogonal projection for $q>1$. We have treated an unsolved case by O'Neil which is $q>1$, and the result that we have obtained is optimal. Second, to investigate a relationship between the multifractal spectrum and its projection onto a lowerdimensional linear subspace. We also obtain general results for the multifractal analysis of the orthogonal projections on $m$-dimensional linear subspaces of a measure $\mu$ satisfying the multifractal formalism.

\section{Preliminaries}

We start by recalling the multifractal formalism introduced by O'Neil [28]. Let $\mu$ be a compactly supported probability measure on $\mathbb{R}^{n}$. For $q, s \in \mathbb{R}, E \subseteq \operatorname{supp} \mu$ and $\delta>0$, we define the multifractal packing pre-measure:

$$
\overline{\mathscr{P}}_{\mu, \delta}^{q, s}(E)=\sup \left\{\sum_{i} \mu\left(B\left(x_{i}, \frac{r_{i}}{3}\right)\right)^{q} r_{i}^{s}\right\},
$$

where the supremum is taken over all $\delta$-packings of $E$ : 


$$
\overline{\mathscr{P}}_{\mu}^{q, s}(E)=\inf _{\delta>0} \overline{\mathscr{P}}_{\mu, \delta}^{q, s}(E) .
$$

The function $\overline{\mathscr{P}}_{\mu}^{q, s}$ is increasing but not $\sigma$-subadditive. That is the reason why O'Neil introduced the modification of the multifractal packing measure $\mathscr{P}_{\mu}^{q, s}$ :

$$
\mathscr{P}_{\mu}^{q, s}(E)=\inf _{E \subseteq \bigcup_{i} E_{i}} \sum_{i} \overline{\mathscr{P}}_{\mu}^{q, s}\left(E_{i}\right)
$$

In a similar way, we define the Hausdorff measure:

$$
\mathscr{H}_{\mu, \delta}^{q, s}(E)=\inf \left\{\sum_{i} \mu\left(B\left(x_{i}, 3 r_{i}\right)\right)^{q} r_{i}^{s} ; \quad\left(B\left(x_{i}, r_{i}\right)\right)_{i} \text { is a } \delta \text {-covering of } E\right\}
$$

and

$$
\mathscr{H}_{\mu}^{q, s}(E)=\sup _{\delta>0} \mathscr{H}_{\mu, \delta}^{q, s}(E) .
$$

The functions $\mathscr{P}_{\mu}^{q, s}$ and $\mathscr{H}_{\mu}^{q, s}$ are metric outer measures and thus measures on the family of Borel subsets of $\mathbb{R}^{n}$. An important feature of the pre-packing, packing and Hausdorff measure is that $\mathscr{P}_{\mu}^{q, s} \leq \overline{\mathscr{P}}_{\mu}^{q, s}$, and there is a constant $c$ depending also on the dimension of the ambient space, such that $\mathscr{H}_{\mu}^{q, s} \leq c \mathscr{P}_{\mu}^{q, s}$ (see [28]).

The functions $\overline{\mathscr{P}}_{\mu}^{q, s}, \mathscr{P}_{\mu}^{q, s}$ and $\mathscr{H}_{\mu}^{q, s}$ assign, in the usual way, a dimension to each subset $E$ of supp $\mu$. They are, respectively, denoted by $\Lambda_{\mu}^{q}(E), B_{\mu}^{q}(E)$ and $b_{\mu}^{q}(E)$.

1. There exists a unique number $\Lambda_{\mu}^{q}(E) \in[-\infty,+\infty]$ such that

$$
\overline{\mathscr{P}}_{\mu}^{q, s}(E)=\left\{\begin{array}{lll}
\infty & \text { if } \quad & s<\Lambda_{\mu}^{q}(E), \\
0 & \text { if } \quad \Lambda_{\mu}^{q}(E)<s .
\end{array}\right.
$$

2. There exists a unique number $B_{\mu}^{q}(E) \in[-\infty,+\infty]$ such that

$$
\mathscr{P}_{\mu}^{q, s}(E)=\left\{\begin{array}{lll}
\infty & \text { if } \quad & s<B_{\mu}^{q}(E), \\
0 & \text { if } \quad & B_{\mu}^{q}(E)<s .
\end{array}\right.
$$

3. There exists a unique number $b_{\mu}^{q}(E) \in[-\infty,+\infty]$ such that

$$
\mathscr{H}_{\mu}^{q, s}(E)=\left\{\begin{array}{lll}
\infty & \text { if } & s<b_{\mu}^{q}(E), \\
0 & \text { if } \quad & b_{\mu}^{q}(E)<s .
\end{array}\right.
$$

We note that for all $q \in \mathbb{R}$

$$
b_{\mu}^{q}(\emptyset)=B_{\mu}^{q}(\emptyset)=\Lambda_{\mu}^{q}(\emptyset)=-\infty,
$$

and if $\mu(E)=0$, then

$$
b_{\mu}^{q}(E)=B_{\mu}^{q}(E)=\Lambda_{\mu}^{q}(E)=-\infty \text { for } q>0 .
$$

Next, we define the separator functions $\Lambda_{\mu}, B_{\mu}$ and $b_{\mu}: \mathbb{R} \rightarrow[-\infty,+\infty]$ by

$$
\Lambda_{\mu}(q)=\Lambda_{\mu}^{q}(\operatorname{supp} \mu), B_{\mu}(q)=B_{\mu}^{q}(\operatorname{supp} \mu) \text { and } b_{\mu}(q)=b_{\mu}^{q}(\operatorname{supp} \mu) \text {. }
$$

It is well known that the functions $\Lambda_{\mu}, B_{\mu}$ and $b_{\mu}$ are decreasing. The functions $\Lambda_{\mu}$ and $B_{\mu}$ are convex and satisfying $b_{\mu} \leq B_{\mu} \leq \Lambda_{\mu}$. 
Proposition 2.1 [28] Let $\mu$ be compactly supported probability measure on $\mathbb{R}^{n}$. Then, we have

1. For $q<1,0 \leq b_{\mu}(q) \leq B_{\mu}(q) \leq \Lambda_{\mu}(q)$.

2. $b_{\mu}(1)=B_{\mu}(1)=\Lambda_{\mu}(1)=0$.

3. For $q>1, b_{\mu}(q) \leq B_{\mu}(q) \leq \Lambda_{\mu}(q) \leq 0$.

Remark 2.1 The multifractal Hausdorff and packing measures introduced by O'Neil are different from those developed by Olsen [26], although, when $\mu$ satisfies a doubling condition, the multifractal measures are equivalent.

\section{Main result}

Let $\mu$ be a compactly supported probability measure on $\mathbb{R}^{n}$ and $q \in \mathbb{R}$. In the following, we require an alternative characterization of the generalized upper $L^{q}$-spectrum of $\mu$ in terms of a potential obtained by convolving $\mu$ with a certain kernel. For this purpose, let us introduce some notations. For $1 \leq s \leq n$ and $r>0$, we define

$$
\begin{aligned}
\phi_{r}^{s}: \mathbb{R}^{n} & \longrightarrow \mathbb{R} \\
x & \longmapsto \min \left\{1, r^{s}|x|^{-s}\right\}
\end{aligned}
$$

and

$$
\mu * \phi_{r}^{s}(x)=\int \min \left\{1, r^{s}|x-y|^{-s}\right\} \mathrm{d} \mu(y) .
$$

Let $E$ be a compact subset of $\operatorname{supp} \mu$. For $1 \leq s \leq n$ and $q>1$, write

$$
N_{\mu, r}^{q, s}(E)=\int_{E}\left(\mu * \phi_{r / 3}^{s}(x)\right)^{q-1} \mathrm{~d} \mu(x)
$$

and

$$
\bar{\tau}_{\mu}^{q, s}(E)=\limsup _{r \rightarrow 0} \frac{\log N_{\mu, r}^{q, s}(E)}{-\log r} \text { and } \underline{\tau}_{\mu}^{q, s}(E)=\liminf _{r \rightarrow 0} \frac{\log N_{\mu, r}^{q, s}(E)}{-\log r} .
$$

The definition of these dimensions is, frankly, messy, indirect and unappealing. In an attempt to make the concept more attractive, we present here an alternative approach to the dimension $\bar{\tau}_{\mu}^{q, s}$ and his application to projections in terms of a potential obtained by convolving $\mu$ with a certain kernel. For $E$ a compact subset of supp $\mu$, we can try to decompose $E$ into a countable number of pieces $E_{1}, E_{2}, \ldots$ in such a way that the largest piece has as small a dimension as possible. The present approach was first used by Falconer [10, Section 3.3] and further developed by O'Neil [28, Proposition 2.4]. This idea leads to the following modified dimension in terms of the convolutions:

$$
\mathfrak{T}_{\mu}^{q, s}(E)=\inf \left\{\sup _{1 \leq i<\infty} \bar{\tau}_{\mu}^{q, s}\left(E_{i}\right), \quad E \subset \bigcup_{i} E_{i} \text { with each } E_{i} \text { compact }\right\}
$$

and

$$
\mathfrak{T}_{\mu}^{s}(q)=\mathfrak{T}_{\mu}^{q, s}(\operatorname{supp} \mu) \text { for all } s \geq 1 .
$$

Let $m$ be an integer with $0<m \leq n$ and $G_{n, m}$ the Grassmannian manifold of all $m$ dimensional linear subspaces of $\mathbb{R}^{n}$. Denote by $\gamma_{n, m}$ the invariant Haar measure on $G_{n, m}$ such that $\gamma_{n, m}\left(G_{n, m}\right)=1$. For $V \in G_{n, m}$, we define the projection map $\pi_{V}: \mathbb{R}^{n} \longrightarrow V$ 
as the usual orthogonal projection onto $V$. Then, the set $\left\{\pi_{V}, V \in G_{n, m}\right\}$ is compact in the space of all linear maps from $\mathbb{R}^{n}$ to $\mathbb{R}^{m}$ and the identification of $V$ with $\pi_{V}$ induces a compact topology for $G_{n, m}$. Also, for a Borel probability measure $\mu$ with compact support supp $\mu \subset \mathbb{R}^{n}$ and for $V \in G_{n, m}$, we denote by $\mu_{V}$, the projection of $\mu$ onto $V$, i.e.,

$$
\mu_{V}(A)=\mu\left(\pi_{V}^{-1}(A)\right) \quad \forall A \subseteq V .
$$

Since $\mu$ is compactly supported and $\operatorname{supp} \mu_{V}=\pi_{V}(\operatorname{supp} \mu)$ for all $V \in G_{n, m}$, for any continuous function $f: V \longrightarrow \mathbb{R}$, we have

$$
\int_{V} f \mathrm{~d} \mu_{V}=\int f\left(\pi_{V}(x)\right) \mathrm{d} \mu(x),
$$

whenever these integrals exist. Then, for all $V \in G_{n, m}, x \in \mathbb{R}^{n}$ and $0<r<1$, we have

$$
\mu * \phi_{r}^{m}(x)=\int \mu_{V}\left(B\left(x_{V}, r\right)\right) \mathrm{d} V=\int \min \left\{1, r^{m}|x-y|^{-m}\right\} \mathrm{d} \mu(y) .
$$

In [28], O'Neil has compared the generalized Hausdorff and packing dimensions of a set $E$ of $\mathbb{R}^{n}$ with respect to a measure $\mu$ with those of their projections onto $m$-dimensional subspaces. More specifically, he proved the following result:

Theorem 3.1 Let $\mu$ be a compactly supported probability measure on $\mathbb{R}^{n}$ and $E \subseteq \operatorname{supp} \mu$. For $q \leq 1$ and all $V \in G_{n, m}$, we have

$$
B_{\mu_{V}}^{q}\left(\pi_{V}(E)\right) \leq B_{\mu}^{q}(E) .
$$

In this paper, we show that $B_{\mu}^{q}(E)$ is preserved under $\gamma_{n, m}$-almost every orthogonal projection for $q>1$. We have treated an unsolved case by O'Neil which is $q>1$, and the result that we have obtained is optimal. More precisely, we have the following result.

Theorem 3.2 Let $E$ be a compact subset of $\operatorname{supp} \mu$ and $q>1$.

1. If $1<q \leq 2$, one has

$$
B_{\mu_{V}}^{q}\left(\pi_{V}(E)\right)=\mathfrak{T}_{\mu}^{q, m}(E)=\max \left(m(1-q), B_{\mu}^{q}(E)\right), \text { for } \gamma_{n, m} \text {-almost every } V \in G_{n, m} .
$$

2. If $q>2$ and $\left(E_{i}\right)_{i}$ is a cover of $E$ by a countable collection of compact sets is such that $\Lambda_{\mu}^{q}\left(E_{i}\right) \geq-m$ for all $i$, then

$$
B_{\mu_{V}}^{q}\left(\pi_{V}(E)\right)=\mathfrak{T}_{\mu}^{q, m}(E)=B_{\mu}^{q}(E), \text { for } \gamma_{n, m} \text {-almost every } V \in G_{n, m} .
$$

As a consequence, we have the following corollary

Corollary 3.1 Let $q>1$.

1. If $1<q \leq 2$, one has

$$
B_{\mu_{V}}(q)=\mathfrak{T}_{\mu}^{m}(q)=\max \left(m(1-q), B_{\mu}(q)\right), \text { for } \gamma_{n, m} \text {-almost every } V \in G_{n, m} .
$$

2. If $q>2$ and $\left(E_{i}\right)_{i}$ is a cover of supp $\mu$ by a countable collection of compact sets is such that $\Lambda_{\mu}^{q}\left(E_{i}\right) \geq-m$ for all $i$, then

$$
B_{\mu_{V}}^{q}(q)=\mathfrak{T}_{\mu}^{m}(q)=B_{\mu}(q), \text { for } \gamma_{n, m} \text {-almost every } V \in G_{n, m} .
$$

Remark 3.1 The hypothesis $\Lambda_{\mu}^{q}\left(E_{i} \cap E\right) \geq-m$ for all $i$ implies that $\Lambda_{\mu}^{q}(E) \geq-m$. Nevertheless, we don't know if the weaker condition $\Lambda_{\mu}^{q}(E) \geq-m$ is sufficient to obtain the conclusion of Theorem 3.2. 


\section{Proof of the main result}

\subsection{Preliminary results}

We present the tools, as well as the intermediate results, which will be used in the proof of our main result. Let $\mu$ be a compactly supported probability measure on $\mathbb{R}^{n}$ and $q \in \mathbb{R}$. We define the upper and lower $L^{q}$ - spectrum of a measure $\mu$. For a subset $E \subset \operatorname{supp} \mu$, write

$$
N_{\mu, r}^{q}(E)=\sup \left\{\sum_{i} \mu\left(B\left(x_{i}, \frac{r}{3}\right)\right)^{q} ; \quad\left(B\left(x_{i}, r\right)\right)_{i} \text { is a packing of } E\right\} .
$$

The upper, respectively, lower $L^{q}$ - spectrum $\bar{\tau}_{\mu}^{q}$ and $\underline{\tau}_{\mu}^{q}$ of $E$ is defined by

$$
\bar{\tau}_{\mu}^{q}(E)=\limsup _{r \rightarrow 0} \frac{\log N_{\mu, r}^{q}(E)}{-\log r} \text { and } \underline{\tau}_{\mu}^{q}(E)=\liminf _{r \rightarrow 0} \frac{\log N_{\mu, r}^{q}(E)}{-\log r} .
$$

By convention, if $r \in(0,1): \frac{\log 0}{-\log r}=-\infty$.

The following proposition is a consequence of the multifractal formalism developed in [28].

Proposition 4.1 Let E be a compact subset of $\operatorname{supp} \mu$ and $q \in \mathbb{R}$. One has

$$
B_{\mu}^{q}(E)=\inf \left\{\sup _{1 \leq i<\infty} \bar{\tau}_{\mu}^{q}\left(E_{i}\right), \quad E \subset \bigcup_{i} E_{i} \text { with each } E_{i} \text { compact }\right\} .
$$

Proposition 4.1 is a consequence from the following lemmas.

Lemma 4.1 Let $E$ be a subset of $\operatorname{supp} \mu$ and $q \in \mathbb{R}$. Then, we have

$$
B_{\mu}^{q}(E)=\inf \left\{\sup _{1 \leq i<\infty} \bar{\tau}_{\mu}^{q}\left(E_{i}\right), E \subset \bigcup_{i} E_{i}\right\}=\inf \left\{\sup _{1 \leq i<\infty} \Lambda_{\mu}^{q}\left(E_{i}\right), E \subset \bigcup_{i} E_{i}\right\} .
$$

Proof The lemma is Proposition 2.4 of [28].

Lemma 4.2 1. For $q, s \in \mathbb{R}$, we have $\overline{\mathscr{P}}_{\mu}^{q, s}(E)=\overline{\mathscr{P}}_{\mu}^{q, s}(\bar{E})$ and $\Lambda_{\mu}^{q}(E)=\Lambda_{\mu}^{q}(\bar{E})$ for all $E \subset \operatorname{supp} \mu$.

2. Let $E$ be a compact subset of $\operatorname{supp} \mu$ and $q \in \mathbb{R}$. If

$$
\Lambda_{\mu}^{q}(E \cap V)=\Lambda_{\mu}^{q}(E) \text { for all open sets } V \text { with } E \cap V \neq \emptyset,
$$

then

$$
B_{\mu}^{q}(E)=\Lambda_{\mu}^{q}(E)
$$

Proof The first part is Lemma 5.4.1 in [25]. We will prove the second part. Let $E \subset \cup_{i} E_{i}$. Since $E \subset \cup_{i} \bar{E}_{i}$, Baire's category theorem implies that there exists an integer $k \in \mathbb{N}$ and an open set $V$ such that $\emptyset \neq E \cap V \subset \bar{E}_{k}$. Hence,

$$
\sup _{i} \Lambda_{\mu}^{q}\left(\bar{E}_{i}\right) \geq \Lambda_{\mu}^{q}\left(\bar{E}_{k}\right) \geq \Lambda_{\mu}^{q}(E \cap V)=\Lambda_{\mu}^{q}(E) .
$$

Since the covering $\left(E_{i}\right)_{i}$ of $E$ was arbitrary, the previous lemma now implies that 


$$
\begin{aligned}
B_{\mu}^{q}(E) & =\inf \left\{\sup _{1 \leq i<\infty} \Lambda_{\mu}^{q}\left(E_{i}\right), \quad E \subset \bigcup_{i} E_{i}\right\} \\
& =\inf \left\{\sup _{1 \leq i<\infty} \Lambda_{\mu}^{q}\left(\overline{E_{i}}\right), \quad E \subset \bigcup_{i} E_{i}\right\} \\
& \geq \Lambda_{\mu}^{q}(E) .
\end{aligned}
$$

The following straightforward estimates concern the behavior of $\mu * \phi_{r}^{n}(x)$ as $r \rightarrow 0$.

Lemma 4.3 [13] Let $1 \leq m \leq n$ and $\mu$ be a compactly supported probability measure on $\mathbb{R}^{n}$. For all $x \in \mathbb{R}^{n}$, we have

$$
c r^{m} \leq \mu * \phi_{r}^{m}(x)
$$

for all sufficiently small $r$, where $c>0$ is independent of $r$.

Lemma 4.4 [13] Let $\mu$ be a compactly supported probability measure on $\mathbb{R}^{n}$.

1. For all $x \in \mathbb{R}^{n}$ and $r>0$,

$$
\mu(B(x, r)) \leq \mu * \phi_{r}^{n}(x) .
$$

2. Let $\varepsilon>0$. We have that for $\mu$-almost all $x$

$$
r^{-\varepsilon} \mu(B(x, r)) \geq \mu * \phi_{r}^{n}(x),
$$

if $r$ is sufficiently small.

We use the properties of $\mu * \phi_{r}^{n}(x)$ to have a relationship between the kernels and projected measures.

Lemma 4.5 [13] Let $1 \leq m \leq n$, $\mu$ be a compactly supported probability measure on $\mathbb{R}^{n}$, $\varepsilon>0$ and $r$ is sufficiently small.

1. For all $V \in G_{n, m}$ and for $\mu$-almost all $x \in \mathbb{R}^{n}$,

$$
r^{\varepsilon} \mu * \phi_{r}^{m}(x) \leq \mu_{V}\left(B\left(x_{V}, r\right)\right) .
$$

2. For $\gamma_{n, m}$-almost all $V \in G_{n, m}$ and all $x \in \mathbb{R}^{n}$,

$$
r^{-\varepsilon} \mu * \phi_{r}^{m}(x) \geq \mu_{V}\left(B\left(x_{V}, r\right)\right) .
$$

The next result is essentially a restatement of [2, Proposition 4.2] and [6, Proposition 5.1] (see also [13, Lemma 2.6 (a)] and [34]). We provide a proof for the reader's convenience.

Proposition 4.2 Let $E$ be a compact subset of $\operatorname{supp} \mu$. For $q>1$, we have

$$
\underline{\tau}_{\mu}^{q}(E)=\liminf _{r \rightarrow 0} \frac{1}{-\log r} \log \int_{E}(\mu(B(x, r / 3)))^{q-1} \mathrm{~d} \mu(x)
$$

and

$$
\bar{\tau}_{\mu}^{q}(E)=\limsup _{r \rightarrow 0} \frac{1}{-\log r} \log \int_{E}(\mu(B(x, r / 3)))^{q-1} \mathrm{~d} \mu(x) .
$$


Proof Let $r>0$ and $\left(B\left(x_{i}, r / 3\right)\right)_{i}$ be a family of disjoint balls centered on $E$. Since $q>1$, we have

$$
\begin{aligned}
\int_{E}(\mu(B(x, r / 3)))^{q-1} \mathrm{~d} \mu(x) & \geq \int_{\bigcup_{i} B\left(x_{i}, r / 9\right)}(\mu(B(x, r / 3)))^{q-1} \mathrm{~d} \mu(x) \\
& \geq \sum_{i}\left(\mu\left(B\left(x_{i}, r / 9\right)\right)\right)^{q} .
\end{aligned}
$$

On the other hand, for every $r>0$ we can apply Besicovitch's covering theorem to $(B(x, r / 3))_{x \in E}$, to get a positive integer $\xi(n)$ depending on $n$ only, as well as $\mathcal{B}_{1}=$ $\left(B\left(x_{1, j}, r / 3\right)\right)_{j}, \ldots, \mathcal{B}_{\xi(n)}=\left(B\left(x_{\xi(n), j}, r / 3\right)\right)_{j}, \xi(n)$ families of disjoint balls of radius $r / 3$, such that $E \subset \bigcup_{i=1}^{\xi(n)} \bigcup_{j} B\left(x_{i, j}, r / 3\right)$ and

$$
\begin{aligned}
\int_{E}(\mu(B(x, r / 9)))^{q-1} \mathrm{~d} \mu(x) & \leq \sum_{i=}^{\xi(n)} \sum_{j} \int_{B\left(x_{i, j}, r / 3\right)}(\mu(B(x, r / 9)))^{q-1} \mathrm{~d} \mu(x) \\
& \leq \sum_{i=}^{\xi(n)} \sum_{j}\left(\mu\left(B\left(x_{i, j}, r / 3\right)\right)\right)^{q} .
\end{aligned}
$$

Taking the logarithms and letting $r \rightarrow 0$ yield the result.

Remark 4.1 Let $E$ be a compact subset of $\operatorname{supp} \mu$ and $q>1$. It is clear that from Proposition 4.2 and Proposition 2.8 in [13],

$$
\bar{\tau}_{\mu}^{q}(E)=\bar{\tau}_{\mu}^{q, n}(E) \text { and } \underline{\tau}_{\mu}^{q}(E)=\underline{\tau}_{\mu}^{q, n}(E) .
$$

With these definitions, we have the following corollaries.

Corollary 4.1 Let $E$ be a compact subset of $\operatorname{supp} \mu$. For $q>1$ and $1 \leq m \leq n$, we have

$$
\underline{\tau}_{\mu}^{q, m}(E)=\inf \left\{s \geq 0 ; \limsup _{r \rightarrow 0} r^{s} \int_{E}\left(\mu * \phi_{r / 3}^{m}(x)\right)^{q-1} \mathrm{~d} \mu(x)=0\right\}
$$

and

$$
\bar{\tau}_{\mu}^{q, m}(E)=\inf \left\{s \geq 0 ; \quad \liminf _{r \rightarrow 0} r^{s} \int_{E}\left(\mu * \phi_{r / 3}^{m}(x)\right)^{q-1} \mathrm{~d} \mu(x)=0\right\} .
$$

Corollary 4.2 Let $E$ be a compact subset of $\operatorname{supp} \mu$. For $q>1$, we have

$$
\underline{\tau}_{\mu}^{q}(E)=\inf \left\{s \geq 0 ; \limsup _{r \rightarrow 0} r^{s} \int_{E}(\mu(B(x, r / 3)))^{q-1} \mathrm{~d} \mu(x)=0\right\}
$$

and

$$
\bar{\tau}_{\mu}^{q}(E)=\inf \left\{s \geq 0 ; \quad \liminf _{r \rightarrow 0} r^{s} \int_{E}(\mu(B(x, r / 3)))^{q-1} \mathrm{~d} \mu(x)=0\right\} .
$$

Proposition 4.3 Let E be a compact subset of $\operatorname{supp} \mu$. For $q>1$, we have

$$
\bar{\tau}_{\mu}^{q, m}(E)=\max \left(m(1-q), \bar{\tau}_{\mu}^{q}(E)\right) .
$$


Proof Recalling that, from Lemma 4.4

$$
\mu * \phi_{r / 3}^{m}(x) \geq \mu(B(x, r / 3)),
$$

it will be clear that for $q>1$ we have

$$
\int_{E}\left(\mu * \phi_{r / 3}^{m}(x)\right)^{q-1} \mathrm{~d} \mu(x) \geq \int_{E}(\mu(B(x, r / 3)))^{q-1} \mathrm{~d} \mu(x) .
$$

Hence,

$$
\underline{\tau}_{\mu}^{q, m}(E) \geq \underline{\tau}_{\mu}^{q}(E) \text { and } \bar{\tau}_{\mu}^{q, m}(E) \geq \bar{\tau}_{\mu}^{q}(E) .
$$

Without loss of generality, we may assume that $\mu(E)>0$. From Lemma 4.3, we get

$$
\int_{E}\left(\mu * \phi_{r / 3}^{n}(x)\right)^{q-1} \mathrm{~d} \mu(x) \geq c_{1} r^{m(q-1)} \mu(E) .
$$

Therefore, we obtain

$$
\underline{\tau}_{\mu}^{q, m}(E) \geq m(1-q) .
$$

Let $t>\bar{\tau}_{\mu}^{q}(E)$. Suppose that $\operatorname{supp} \mu$ have diameter $h$. Then,

$$
\int_{E}(\mu(B(x, r / 3)))^{q-1} \mathrm{~d} \mu(x) \leq c_{1} r^{-t}, \quad \forall r \leq 6 h,
$$

where $c_{1}$ is independent of $r$, and

$$
\int_{E}(\mu(B(x, r / 3)))^{q-1} \mathrm{~d} \mu(x)=1, \quad \forall r \geq 6 h .
$$

For $\varepsilon>0, p=q-1$ and $r$ is small enough, by using Proposition 2.5 in [13], we obtain

$$
\begin{aligned}
\int_{E}\left(\mu * \phi_{r / 3}^{m}(x)\right)^{p} \mathrm{~d} \mu(x) \leq & B(r / 3)^{m p-\varepsilon} \int_{r / 3}^{+\infty} u^{-m q-1} \int_{E}(\mu(B(x, u)))^{p} \mathrm{~d} \mu(x) \mathrm{d} u \\
= & B(r / 3)^{m p-\varepsilon} \int_{r / 3}^{6 h} u^{-m q-1} \int_{E}(\mu(B(x, u)))^{p} \mathrm{~d} \mu(x) \mathrm{d} u \\
& +B(r / 3)^{m p-\varepsilon} \int_{6 h}^{+\infty} u^{-m p-1} \int_{E}(\mu(B(x, u)))^{p} \mathrm{~d} \mu(x) \mathrm{d} u \\
\leq & C_{1}(r / 3)^{m p-\varepsilon} \int_{r / 3}^{6 h} u^{-m p-1-t} \mathrm{~d} u \\
& +C_{2}(r / 3)^{m p-\varepsilon} \int_{6 h}^{+\infty} u^{-m p-1} \mathrm{~d} u \\
\leq & \begin{cases}C_{3}(r / 3)^{-t-\varepsilon} & \text { si } t>-m p, \\
C_{4}(r / 3)^{m p-\varepsilon} \text { si } t \leq-m p,\end{cases}
\end{aligned}
$$

where $B$ and $C_{i}(i=1, \ldots, 4)$ are independent of $r$. This gives that

$$
\bar{\tau}_{\mu}^{q, m}(E) \leq \max (-m p, t), \text { for all } t>\bar{\tau}_{\mu}^{q}(E) .
$$

Finally, we obtain

$$
\bar{\tau}_{\mu}^{q, m}(E) \leq \max \left(m(1-q), \bar{\tau}_{\mu}^{q}(E)\right) .
$$


The following results present alternative expressions of the $L^{q}$-spectrum in terms of the convolutions as well as general relations between the $L^{q}$-spectrum of a measure and that of its orthogonal projections.

Theorem 4.1 Let $E$ be a compact subset of supp $\mu$. Then, we have

1. For all $q>1$ and $V \in G_{n, m}$,

$$
\underline{\tau}_{\mu_{V}}^{q}\left(\pi_{V}(E)\right) \geq \underline{\tau}_{\mu}^{q, m}(E) \text { and } \bar{\tau}_{\mu_{V}}^{q}\left(\pi_{V}(E)\right) \geq \bar{\tau}_{\mu}^{q, m}(E) .
$$

2. For all $1<q \leq 2$ and $\gamma_{n, m}$-almost every $V \in G_{n, m}$,

$$
\bar{\tau}_{\mu_{V}}^{q}\left(\pi_{V}(E)\right)=\bar{\tau}_{\mu}^{q, m}(E)=\max \left(m(1-q), \bar{\tau}_{\mu}^{q}(E)\right)
$$

and

$$
\underline{\tau}_{\mu_{V}}^{q}\left(\pi_{V}(E)\right)=\underline{\tau}_{\mu}^{q, m}(E) .
$$

3. For all $q>2$ and $\gamma_{n, m}$-almost every $V \in G_{n, m}$,

(a) If $-m \leq \bar{\tau}_{\mu}^{q}(E)$ then $\bar{\tau}_{\mu_{V}}^{q}\left(\pi_{V}(E)\right)=\bar{\tau}_{\mu}^{q, m}(E)=\bar{\tau}_{\mu}^{q}(E)$.

(b) $\underline{\tau}_{\mu_{V}}^{q}\left(\pi_{V}(E)\right)=\max \left(m(1-q), \underline{\tau}_{\mu}^{q, m}(E)\right)$.

Remark 4.2 The assertion (2) is essentially a restatement of the main result of Hunt and Kaloshin [17] and Falconer and O'Neil [13, Theorem 3.9]. The assertion (3) extends the result of Hunt and Kaloshin (of Falconer and O'Neil) to the case $q>2$ untreated in their work.

Proof The first and second parts follow from Proposition 4.3 and the following lemma which is a consequence of Lemma 4.5.

Lemma 4.6 Let $E$ be a compact subset of supp $\mu$. Then, we have

1. for all $q>1$ and $V \in G_{n, m}$,

$$
\limsup _{r \rightarrow 0} \frac{1}{-\log r}\left[\log \left(\frac{\int_{E}\left(\mu * \phi_{r / 3}^{n}(x)\right)^{q-1} \mathrm{~d} \mu(x)}{\int_{\pi_{V}(E)}\left(\mu_{V}\left(B\left(x_{V}, r / 3\right)\right)\right)^{q-1} \mathrm{~d} \mu_{V}\left(x_{V}\right)}\right)\right] \leq 0,
$$

2. for $1<q \leq 2$ and $\gamma_{n, m}$-almost every $V \in G_{n, m}$,

$$
\lim _{r \rightarrow 0} \frac{1}{-\log r}\left[\log \left(\frac{\int_{E}\left(\mu * \phi_{r / 3}^{n}(x)\right)^{q-1} \mathrm{~d} \mu(x)}{\int_{\pi_{V}(E)}\left(\mu_{V}\left(B\left(x_{V}, r / 3\right)\right)\right)^{q-1} \mathrm{~d} \mu_{V}\left(x_{V}\right)}\right)\right]=0 .
$$

See [1, Theorem 2.1], [6, Theorem 4.1] and [31] for the key ideas needed to prove the third part of Theorem 4.1. 


\subsection{Proof of Theorem 3.2}

Let us prove our main theorem. Let $q>1$.

1. If $s>\mathfrak{T}_{\mu}^{q, m}(E)$, we may cover $E$ by a countable collection of sets $E_{i}$, which we may take is compact, such that $\bar{\tau}_{\mu}^{q, m}\left(E_{i}\right)<s$. By using Theorem 4.1 (2.), we have $\bar{\tau}_{\mu_{V}}^{q}\left(\pi_{V}\left(E_{i}\right)\right) \leq$ $s$ for $\gamma_{n, m}$-almost every $V \in G_{n, m}$. Proposition 4.1 implies that $B_{\mu_{V}}^{q}\left(\pi_{V}(E)\right) \leq s$ for $\gamma_{n, m}$-almost every $V \in G_{n, m}$, and so, $B_{\mu_{V}}^{q}\left(\pi_{V}(E)\right) \leq \mathfrak{T}_{\mu}^{q, m}(E)$ for $\gamma_{n, m}$-almost every $V \in G_{n, m}$.

Now, if $s<\mathfrak{T}_{\mu}^{q, m}(E)$. Fix $V \in G_{n, m}$ and let $\left(\widetilde{E}_{i}\right)_{i}$ be a cover of the compact set $\pi_{V}(E)$ by a countable collection of compact sets. Put for each $i, E_{i}=E \cap \pi_{V}^{-1}\left(\widetilde{E}_{i}\right)$, then $\sup _{i} \bar{\tau}_{\mu}^{q, m}\left(E_{i}\right)>s$. By using Theorem 4.1 (1.), we have $\sup _{i} \bar{\tau}_{\mu_{V}}^{q}\left(\pi_{V}\left(E_{i}\right)\right) \geq$ $s$ and $\sup _{i} \bar{\tau}_{\mu_{V}}^{q}\left(\widetilde{E}_{i}\right) \geq s$, this implies that $B_{\mu_{V}}^{q}\left(\pi_{V}(E)\right) \geq s$. Therefore, we obtain $B_{\mu_{V}}^{q}\left(\pi_{V}(E)\right) \geq \mathfrak{T}_{\mu}^{q, m}(E)$.

Thus, the part concerning the equality between $\max \left(m(1-q), B_{\mu}^{q}(E)\right)$ and $\mathfrak{T}_{\mu}^{q, m}(E)$ is a consequence of Proposition 4.3.

2. Let $\left(E_{i}\right)_{i}$ be a cover of $E$ by a countable collection of compact sets is such that $\bar{\tau}_{\mu}^{q}\left(E_{i}\right)=$ $\Lambda_{\mu}^{q}\left(E_{i}\right) \geq-m$ for all $i$. Then, by using Lemma 4.1 and Proposition 4.3 , we have $\mathfrak{T}_{\mu}^{q, m}(E)=B_{\mu}^{q}(E)$.

Now, if $s>\mathfrak{T}_{\mu}^{q, m}(E)$, we may cover $E$ by a countable collection of sets $E_{i}$, which we may take to be compact, such that $\bar{\tau}_{\mu}^{q, m}\left(E_{i}\right)<s$. By using Theorem 4.1 (3.) and since $-m \leq \bar{\tau}_{\mu}^{q}\left(E_{i}\right)=\Lambda_{\mu}^{q}\left(E_{i}\right)$ for all $i$, we have $\bar{\tau}_{\mu_{V}}^{q}\left(\pi_{V}\left(E_{i}\right)\right) \leq s$ for $\gamma_{n, m}$-almost every $V \in G_{n, m}$. Proposition 4.1 implies that $B_{\mu_{V}}^{q}\left(\pi_{V}(E)\right) \leq s$ for $\gamma_{n, m}$-almost every $V \in G_{n, m}$, and so, $B_{\mu_{V}}^{q}\left(\pi_{V}(E)\right) \leq \mathfrak{T}_{\mu}^{q, m}(E)$ for $\gamma_{n, m}$-almost every $V \in G_{n, m}$. In similar way, we prove $B_{\mu_{V}}^{q}\left(\pi_{V}(E)\right) \geq \mathfrak{T}_{\mu}^{q, m}(E)$ for all $V \in G_{n, m}$.

We can improve substantially the O'Neil's result [28, Corollary 5.12] in the following example:

Example 4.1 Fix $0<m \leq n$ and let $\mu$ be a self-similar measure on $\mathbb{R}^{n}$ with support equal to $K$ such that $\operatorname{dim}_{P}(K)=s \leq m$. Let $q \geq 0$ and $\left(E_{i}\right)_{i}$ be a cover of $E$ by a countable collection of compact sets such that $\Lambda_{\mu}^{q}\left(E_{i}\right) \geq-m$ for all $i$. By using Corollary 3.1 and Corollary 5.12 in [28], we have for $\gamma_{n, m}$-almost every $V \in G_{n, m}$

$$
B_{\mu_{V}}(q)=b_{\mu_{V}}(q)=b_{\mu}(q)=B_{\mu}(q) \text {. }
$$

\section{Application}

When $\mu$ obeys the multifractal formalism over some interval, we are interested in knowing whether or not this property is preserved after orthogonal projections on $\gamma_{n, m}$-almost every linear $m$-dimensional subspace.

This section is devoted to study the behavior of projections of measures obeying to the multifractal formalism. More precisely, we prove that for $q>1$ if the multifractal formalism holds for $\mu$ at $\alpha=-B_{\mu}^{\prime}(q)$, it holds for $\mu_{V}$ for $\gamma_{n, m}$-almost every $V \in G_{n, m}$. Before detailing our results, let us recall the multifractal formalism introduced by O'Neil. For $\alpha \geq 0$, let

$$
E_{\mu}(\alpha)=\left\{x \in \operatorname{supp} \mu ; \lim _{r \rightarrow 0} \frac{\log (\mu B(x, 3 r))}{\log r}=\alpha\right\} .
$$


We mention that in the last decade there has been a great interest for the multifractal analysis and positive results have been written in various situations (see, for example, [4,5,26,27]).

The function $B_{\mu}(q)$ is related to the multifractal spectrum of the measure $\mu$. More precisely, $f^{*}(\alpha)=\inf _{\beta}(\alpha \beta+f(\beta))$ denotes the Legendre transform of the function $f$; it has been proved in $[4,5,26,27]$ a lower and upper bound estimate of the singularity spectrum using the Legendre transform of the function $B_{\mu}(q)$. The following theorem is a consequence of the multifractal formalism developed in [5].

Theorem 5.1 Let $\mu$ be a compactly supported Borel probability measure on $\mathbb{R}^{n}$ and $q \in \mathbb{R}$. Suppose that

1. $\mathscr{H}_{\mu}^{q, B_{\mu}(q)}(\operatorname{supp} \mu)>0$,

2. $B_{\mu}$ is differentiable at $q$.

Then,

$$
\operatorname{dim}_{H} E_{\mu}\left(-B_{\mu}^{\prime}(q)\right)=\operatorname{dim}_{P} E_{\mu}\left(-B_{\mu}^{\prime}(q)\right)=B_{\mu}^{*}\left(-B_{\mu}^{\prime}(q)\right)=b_{\mu}^{*}\left(-B_{\mu}^{\prime}(q)\right) .
$$

Here $\operatorname{dim}_{H}$ and $\operatorname{dim}_{P}$ denote, respectively, the Hausdorff and the packing dimension, see [26] for precise definitions of this.

The following proposition is established in [28].

Proposition 5.1 Let $\mu$ be a compactly supported Borel probability measure on $\mathbb{R}^{n}$. For $q \geq 1$ and all $V \in G_{n, m}$, we have

$$
b_{\mu_{V}}(q) \geq \max \left(m(1-q), b_{\mu}(q)\right) .
$$

In the following, we study the validity of the multifractal formalism under projection. More specifically, we obtain general result for the multifractal analysis of the orthogonal projections on $m$-dimensional linear subspaces of measure $\mu$ satisfying the multifractal formalism.

Theorem 5.2 Let $\mu$ be a compactly supported Borel probability measure on $\mathbb{R}^{n}$ and $q>1$. Suppose that

$\left(H_{1}\right) \mathscr{H}_{\mu}^{q, B_{\mu}(q)}(\operatorname{supp} \mu)>0$,

$\left(\mathrm{H}_{2}\right) B_{\mu}$ is differentiable at $q$,

$\left(H_{3}\right)\left(E_{i}\right)_{i}$ be a cover of $\operatorname{supp} \mu$ by a countable collection of compact sets is such that $b_{\mu}^{q}\left(E_{i} \cap \operatorname{supp} \mu\right) \geq \max (-m, m(1-q))$ for all $i$.

Then, for $\gamma_{n, m}$-almost every $V \in G_{n, m}$,

$$
\begin{aligned}
\operatorname{dim}_{P} E_{\mu_{V}}\left(-B_{\mu}^{\prime}(q)\right) & =\operatorname{dim}_{H} E_{\mu_{V}}\left(-B_{\mu}^{\prime}(q)\right)=\operatorname{dim}_{H} E_{\mu}\left(-B_{\mu}^{\prime}(q)\right) \\
& =\operatorname{dim}_{P} E_{\mu}\left(-B_{\mu}^{\prime}(q)\right)=B_{\mu}^{*}\left(-B_{\mu}^{\prime}(q)\right)=b_{\mu}^{*}\left(-B_{\mu}^{\prime}(q)\right) .
\end{aligned}
$$

Remark 5.1 The results of Theorem 5.2 hold if we replace the condition

$$
\mathscr{H}_{\mu}^{q, B_{\mu}(q)}(\operatorname{supp} \mu)>0
$$

by the existence of a nontrivial (Frostman) measure $v_{q}$ satisfying

$$
v_{q}(B(x, r)) \leq \mu(B(x, 3 r))^{q} r^{B_{\mu}(q)}
$$

where $x \in \operatorname{supp} \mu$ and $0<r<1$.

For more details, the reader can see [28, Theorem 5.1]. 
Proof By using Corollary 3.1, Proposition 5.1, $\left(\mathrm{H}_{1}\right)$ and $\left(\mathrm{H}_{3}\right)$, we have, for $\gamma_{n, m}$-almost every $V \in G_{n, m}$,

$$
b_{\mu}(q)=b_{\mu_{V}}(q)=B_{\mu_{V}}(q)=B_{\mu}(q) .
$$

$\left(H_{1}\right),(5.1)$ and the proof of Lemma 3.2 in [28] ensure that there exists a positive constant $c$ such that

$$
0<\mathscr{H}_{\mu}^{q, B_{\mu}(q)}(\operatorname{supp} \mu) \leq c \mathscr{H}_{\mu_{V}}^{q, B_{\mu_{V}}(q)}\left(\operatorname{supp} \mu_{V}\right), \quad \text { for } \gamma_{n, m} \text {-almost every } V \in G_{n, m} .
$$

So, the hypothesis $\left(\mathrm{H}_{2}\right)$, Theorem 5.1 and the equalities (5.1) imply that

$$
\operatorname{dim}_{H} E_{\mu_{V}}\left(-B_{\mu}^{\prime}(q)\right) \geq-q B_{\mu}^{\prime}(q)+B_{\mu}(q), \text { for } \gamma_{n, m} \text {-almost every } V \in G_{n, m} \text {. }
$$

Hence, the assumption (5.1) gives that

$$
\begin{aligned}
\operatorname{dim}_{P} E_{\mu_{V}}\left(-B_{\mu}^{\prime}(q)\right) & \leq B_{\mu_{V}}^{*}\left(-B_{\mu}^{\prime}(q)\right) \\
& =B_{\mu}^{*}\left(-B_{\mu}^{\prime}(q)\right),
\end{aligned}
$$

for $\gamma_{n, m}$-almost every $V \in G_{n, m}$. Thus, the result is a consequence from (5.2) and (5.3).

Acknowledgements The author is greatly indebted to the referee for carefully reading the first submitted version of this paper and giving elaborate comments and valuable suggestions on revision so that the presentation can be greatly improved.

\section{References}

1. Bahroun, F., Bhouri, I.: Multifractals and projections. Extr. Math. 21, 83-91 (2006)

2. Barral, J., Bhouri, I.: Multifractal analysis for projections of Gibbs and related measures. Ergod. Theory Dyn. Syst. 31, 673-701 (2011)

3. Barral, J., Feng, D.J.: Projections of planar Mandelbrot random measures. Adv. Math. 325, 640-718 (2018)

4. Barral, J., Ben Nasr, F., Peyrière, J.: Comparing multifractal formalism: the neighbouring box condition. Asian J. Math. 7, 149-166 (2003)

5. Ben Nasr, F., Bhouri, I., Heurteaux, Y.: The validity of the multifractal formalism: results and examples. Adv. Math. 165, 264-284 (2002)

6. Bhouri, I.: On the projections of generalized upper $L^{q}$-spectrum. Chaos Solitons Fractals 42, 1451-1462 (2009)

7. Douzi, Z., Selmi, B.: Multifractal variation for projections of measures. Chaos Solitons Fractals 91, 414-420 (2016)

8. Douzi, Z., Selmi, B.: On the projections of mutual multifractal spectra (2018). arXiv:1805.06866v1

9. Douzi, Z., Selmi, B.: On the projections of the mutual multifractal Rényi dimensions. Anal. Theory Appl. (to appear)

10. Falconer, K.J.: Fractal Geometry: Mathematical Foundations and Applications. Wiley, Chichester (1990)

11. Falconer, K.J., Howroyd, J.D.: Packing dimensions of projections and dimensions profiles. Math. Proc. Camb. Philos. Soc. 121, 269-286 (1997)

12. Falconer, K.J., Mattila, P.: The packing dimensions of projections and sections of measures. Math. Proc. Camb. Philos. Soc. 119, 695-713 (1996)

13. Falconer, K.J., O’Neil, T.C.: Convolutions and the geometry of multifractal measures. Math. Nachr. 204, 61-82 (1999)

14. Falconer, K.J., Mattila, P.: Strong Marstrand theorems and dimensions of sets formed by subsets of hyperplanes. J. Fractal Geom. 3, 319-329 (2016)

15. Falconer, K.J., Jim, X.: Exact dimensionality and projections of random self-similar measures and sets. J. Lond. Math. Soc. 90, 388-412 (2014)

16. Hu, X., Taylor, J.: Fractal properties of products and projections of measures in $\mathbb{R}^{n}$. Math. Proc. Camb. Philos. Soc. 115, 527-544 (1994)

17. Hunt, B.R., Kaloshin, V.Y.: How projections affect the dimension spectrum of fractal measures. Nonlinearity 10, 1031-1046 (1997) 
18. Järvenpää, E., Järvenpä, M.: Linear mappings and generalized upper spectrum for dimensions. Nonlinearity 45, 55-77 (1998)

19. Järvenpää, E., Järvenpä, M., Ledrappier, F., Leikas, M.: One-dimensional families of projections. Nonlinearity 21, 453-464 (2008)

20. Järvenpää, E., Järvenpä, M., Keleti, T.: Hausdorff dimension and non-degenerate families of projections. J. Geom. Anal. 24, 2020-2034 (2014)

21. Kaufman, R.: On Hausdorff dimension of projections. Mathematika 15, 153-155 (1968)

22. Marstrand, J.M.: Some fundamental geometrical properties of plane sets of fractional dimensions. Proc. Lond. Math. Soc. 4, 257-302 (1954)

23. Mattila, P.: Hausdorff dimension, orthogonal projections and intersections with planes. Ann. Acad. Sci. Fenn. Math. 1, 227-244 (1975)

24. Mattila, P.: The Geometry of Sets and Measures in Euclidean Spaces. Cambridge University Press, Cambrdige (1995)

25. Olsen, L.: Multifractal dimensions of product measures. Math. Proc. Camb. Philos. Soc. 120, 709-734 (1996)

26. Olsen, L.: A multifractal formalism. Adv. Math. 166, 82-196 (1995)

27. Olsen, L.: Dimension inequalities of multifractal Hausdorff measures and multifractal packing measures. Math. Scand. 86, 109-129 (2000)

28. O'Neil, T.C.: The multifractal spectra of projected measures in Euclidean spaces. Chaos Solitons Fractals 11, 901-921 (2000)

29. Shmerkin, P.: Projections of self-similar and related fractals: a survey of recent developments. Fractal Geom. Stoch. V 70, 53-74 (2015)

30. Shmerkin, P., Solomya, B.: Absolute continuity of self-similar measures, their projections and convolution. Trans. Am. Math. Soc. 368, 5125-5151 (2016)

31. Selmi, B.: A note on the effect of projections on both measures and the generalization of $q$-dimension capacity. Probl. Anal. Issues Anal. 5(23), 38-51 (2016)

32. Selmi, B.: On the effect of projections on the Billingsley dimensions. Asian-Eur. J. Math. 13, 2050128, 1-17 (2020)

33. Selmi, B.: Multifractal dimensions for projections of measures. Bol. Soc. Paran. Mat. (2019). https://doi. org/10.5269/bspm.44913

34. Selmi, B., Svetova, NYu.: On the projections of mutual $L^{q, t}$-spectrum. Probl. Anal. Issues Anal. 6(24), 94-108 (2017)

Publisher's Note Springer Nature remains neutral with regard to jurisdictional claims in published maps and institutional affiliations. 